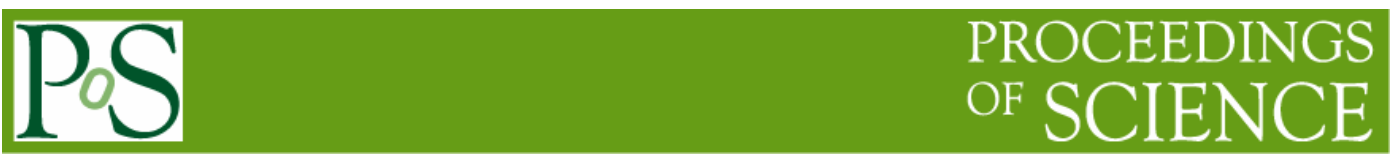

\title{
Performances of digital acquisition for a BC501A detector system
}

\section{Lebreton`, J.F. Guerre-Chaley}

Institute of Radiological Protection and Nuclear Safety (IRSN)

13115 Cadarache, France

E-mail: Iena.lebretoneirsn.fr; jean-francois.guerre-chaleyeirsn.fr

\section{Manuel, B. Duponchel, V. Maury}

University Paris Sud, IUT, 9 avenue de la division Leclerc,

94234 Cachan, France

E-mail: diane.manuel@math.u-cergy.fr

IRSN uses a BC501A detector system to determine reference fluence energy distribution of neutron fields in the energy range between 1 and $20 \mathrm{MeV}$. The data acquisition chain associated to this detector consists of NIM standard electronic units which makes the system bulky and not easily movable. Recent developments in the field of numerical electronics offer the opportunity to use digital acquisition system which is compact, more convenient for measurements at workplaces and allows numerical data treatment.

Within the framework of collaboration between IRSN and the technological university of Cachan a new digital acquisition system was developed and tested with the BC501A. An Acqiris digital card with a data recording capability of 8 bits range at $4 \mathrm{GHz}$ on two channels was chosen. Specific electronic and signal processing were developed to allow gain stability with high voltage feedback, and to obtain energy information with neutron $/ \gamma$ discrimination. The performances (energy resolution, threshold values, dead time and count rate) of the digital acquisition prototype were then compared with those of standard analogical acquisition. In the present state, the comparison results were not satisfying for metrology applications, but the use of new digital cards with 10 bits range is awaited to be fully competitive.

International Workshop on Fast Neutron Detectors

University of Cape Town, South Africa

April 3-6, 2006

\footnotetext{
${ }^{*}$ Speaker 


\section{Introduction}

IRSN uses a liquid scintillator (BC501A, 2"*2") detector system to determine reference fluence energy distribution of neutron fields in the energy range between 1 and $20 \mathrm{MeV}$. Within the framework of collaboration between IRSN and the technological university of Cachan a digital acquisition system was developed and tested for this system.

Several reasons have motivated the study: the actual analogical system based on NIM units, is heavy and brittle as regard of transport vibrations. Because this detector system is set in front of different neutron facilities, reducing the size and the weight of the detector system is an interesting challenge. Moreover the development of such kind of system for outside measurement (medical neutron facilities, nuclear industry) is also an answer for activities of the laboratory. Last, digital electronic improvement offer new ways to acquire pulses from detector system.

Since the development of specific electronic was not possible for budget reasons, a digital unit was chosen from the market. The performances of the new digital acquisition system are compared with the analogical ones.

\section{Description principle of BC501A detector system}

The scintillation liquid cell BC501A (alias NE213) is well known to detect fast neutrons and photons. The fast neutrons and photons create recoil protons and Compton electrons respectively, with energy ranges between 0 and the neutron incident energy or the Compton edge value for the gamma. The time characteristic of the scintillation light is slightly different for electron and recoil proton. This difference is used to discriminate the two types of particles.

Two signals are used from the phototube, the first comes from the anode, the second from the $9^{\text {th }}$ out of 12 dynodes. The fall time of the anodic signal depends on the nature of the secondary charged particle. The dynode signal is related to the secondary charged particle energy.

\section{Description of analogical acquisition}

The analogical acquisition electronic system used is in N.I.M. standard and is mainly constituted of ORTEC and Fast Comptec units. The anode and dynode pulses are transmitted via 60 meters long cables to this DAQ system. The discrimination between the pulses coming from recoil protons and Compton electrons is performed by the use of the zero crossing method on the anode signal.

The height of the dynode signal (after integration and amplification by a pre-amplifier) is related to the secondary charged particle energy. 


\section{Description of digital acquisition}

The choice of the digital unit was constrained by the characteristics of the pulse [5]. The digital unit, chose in 2002, is an Acqiris model DC241. The characteristics of this card are presented in Table 1 .

\begin{tabular}{|l|l|}
\hline Sampling frequency & $\begin{array}{l}4 \mathrm{Gs} / \mathrm{s} \\
\text { Digitalisation of } 4.10^{9} \text { points per second on the pulse }\end{array}$ \\
\hline Number of channel & 2 \\
\hline Band width & $1 \mathrm{GHz}$ \\
\hline Resolution & $8 \mathrm{bits}$ \\
\hline Time stamping & yes \\
\hline Transfer speed & 10000 curves/s \\
\hline Full scale & From $50 \mathrm{mV}$ to 5 Volts \\
\hline
\end{tabular}

Table 1: Characteristics of the digital Acqiris card

This card presents useful characteristics: each pulse recorded is time stamped, that allows acquisition time control; the card can be used near the detector with a connection to a PC with optical fibre (high distance and CEM immunity). The major drawback is the poor resolution limited to 256 channels (8bits) compared with at least 1024 channels (10 bits) available with analogical acquisition. No digital cards offered higher resolution with suitable bandwidth at that time. Choice was done to prioritize the sampling frequency for the first prototype.

The duration of negative anode pulse is between 100 and $150 \mathrm{~ns}$ (depending of energy and kinds of particles). To have similar time characteristics for anode and dynode pulses, dynode pulse (duration $\sim 100 \mathrm{~ns}$ ) is recorded directly from the phototube.

\subsection{Energy determination}

Energy information can be either given by integration of dynode pulse or by the pulse height of anode pulse, but with lower resolution. To control coherence the two ways are computed and compared, but integration of the dynode pulse is only used. An intrinsic threshold, due to Acqiris card design, is fixed to 4 to $6 \%$ of the full scale. In order to acquire the entire pulse and to evaluate the base time, samples are recorded $40 \mathrm{~ns}$ before the trigger. 


\subsection{Neutron-gamma discrimination}

Neutron-gamma discrimination is performed by analysis of the anode pulse (figure 1). The curve can be described by a function $f(t)$. This function is different for neutron $f_{n}(t)$ and gamma $f_{\gamma}(t)$.



Figure 1: Theoretical scheme of anode pulse

The integral of the curve depends of decreasing time (type of particles) and pulse height (energy).The integration of the curve runs during acquisition time T.

$$
\int_{0}^{T} f_{n}(t) \cdot d t \text { or } \int_{0}^{T} f_{\gamma}(t) \cdot d t
$$

The aim was then to define a component independent of the type of particles but dependent of the energy. Subtraction of the two parts of curves enhanced region of differences. The value $t_{0}$ was chosen to have equality between the difference at the right of the threshold and the difference at the left of the threshold:

$$
\int_{0}^{t_{0}}\left(f_{n}(t)-f_{\gamma}(t)\right) \cdot d t=\int_{t_{0}}^{T}\left(f_{n}(t)-f_{\gamma}(t)\right) \cdot d t
$$

If the function $\mathrm{G}(\mathrm{t})$ is defined as:

$G(t)=\int_{0}^{T} f(t) \cdot \Gamma(t) d t$

With: $\quad \Gamma(t)=+1$ for $\mathrm{t}<\mathrm{t}_{0} \quad$ and $\quad \Gamma(t)=-1$ for $\mathrm{t}>\mathrm{t}_{0}$

Then:

$G_{n}(t)=\int_{0}^{T} f_{n}(t) \cdot \Gamma(t) \cdot d t=G_{\gamma}(t)=\int_{0}^{T} f_{\gamma}(t) \cdot \Gamma(t) \cdot d t$

The function $\mathrm{G}(\mathrm{t})$ is then independent of the nature of particles. It depends only of the energy.

And ratio: $\frac{\int_{0}^{T} f_{n}(t) \cdot d t}{\int_{0}^{T}\left(f_{n}(t) \cdot \Gamma(t)\right) \cdot d t}$ or $\frac{\int_{0}^{T} f_{\gamma}(t) \cdot d t}{\int_{0}^{T}\left(f_{\gamma}(t) \cdot \Gamma(t)\right) \cdot d t}$

depends of the nature of particle but are independent of energy of the particles. 
Use of $\Gamma(t)$ function is known as the quarter height method. The process is done in two steps: first determination of $\mathrm{t}_{0}$, which is the time where the curve is reaching down the quarter of its maximum, and then computation of the integrals. This makes the process slow. The choice of $\mathrm{t}_{0}$ satisfying the relation (2) is very sensitive.

Similar results can be obtained with a smooth function like sine to replace the square function; in this case the position of the sign threshold is less critical. The methods using sine function is equivalent to use the Fourier Transform method.

$$
\begin{array}{ll}
F T(f)(0)=\int_{0}^{T} f(t) \cdot d t & (6) \text { is integral of the curve and } \\
F T(f)(\xi)=\int_{0}^{T} f(t) \cdot \sin (2 \pi \xi t) \cdot d t & \text { (7) corresponding to the standard width of the }
\end{array}
$$
curves, then ratio becomes:

$$
K(f)=\frac{|F T(f)(0)|}{|F T(f)(\xi)|}
$$

The best discrimination between the two kinds of particles is obtained for $\xi=\frac{1}{T}$ where

$\mathrm{T}$ is integration duration. Two integrals are computed in parallel, and then one division is performed. Taking absolute values makes the process translation-invariant.

Other methods were tested. Zero crossing and integration of charge methods, commonly used by analogical process, suffering of the lack of points to describe with precision the curves recorded or take to much time to calculate values. Statistical linear regression method or variance method was tested; their use was convenient but more calculations were needed.

\section{Experimental measurements}

Experimental measurements were performed with neutrons (February and March 2006) at the IRSN AMANDE facility [7] with the second IRSN liquid scintillator. This detector system generally used as monitor was placed at 6 meters from the target, at an angular position of 17 degrees with respect to the beam axis. Some measurements were also performed with an ${ }^{88} \mathrm{Y}$ gamma source for evaluation of the resolution with the Compton edges.

\subsection{Recorded data with analogical process and data reduction}

For this monitor detector system the ADC used is FAST TDC-7072 associated to FAST ComTec MPA-3 system. A three dimensional plot (Energy, pulse shape parameter, counts) is used for the analysis of the data. A home made software allows reading the three dimensional plot, separating neutrons and gamma as a function of a separating curve defined "by hand", and projecting neutron or gamma events on the energy axis. A live time taking into account dead time from the ADC and the computer is given by the software. 


\subsection{Recorded data with digital process and data reduction}

The high voltage was delivered by a unit developed by the CRIIP and driven by the computer; the anode and dynode outputs are connected to the Acqiris card and then collected via optical fibre to the computer. The software developed by the CRIIP, allows the reading of the data from the card, then computes energy from dynode pulse and discriminates neutron and gamma with the Fourier Transform method. A three dimensional plot is recorded. The data reduction is done in the same way than for the analogical process. Some spurious events probably generated by the soft were ejected before projection on $\mathrm{X}$ axis. The Acqiris card offer a restrictive choice (7) of full scale calibration factor from $50 \mathrm{mV}$ to $5 \mathrm{~V}$, only three values can be used by the IRSN system: $0.2 \mathrm{~V}, 0.5 \mathrm{~V}$ and $1 \mathrm{~V}$. For a full scale value less than $0.2 \mathrm{~V}$ Compton energy distribution of ${ }^{88} \mathrm{Y}$ is overloaded. The recoil proton energy distribution for 14.75 MeV and $18.8 \mathrm{MeV}$ neutron beam were respectively recorded with full scales of $0.5 \mathrm{~V}$ and $1 \mathrm{~V}$.

\section{Analysis and comparison}

\subsection{Discrimination neutron-gamma with digital process}

The discrimination plot recorded with digital acquisition for neutron beam of $14.75 \mathrm{MeV}$ is shown on figure 2. The discrimination is satisfying: the two clouds of particle are well separated and the value of the discrimination threshold is comparable to the one obtained with the analogical DAQ.



Figure 2: Plot recorded by digital acquisition during measurements with $14.75 \mathrm{MeV}$ neutrons. 


\subsection{Energy resolution}

The recoil proton energy distributions obtained with $14.75 \mathrm{MeV}$ and $18.8 \mathrm{MeV}$ neutron beams were compared with results of the NRESP[3] code. As shown on figure 3, experimental data are in agreement with the calculation. For high neutron energy a relatively good agreement is obtained but further measurements are needed to confirm this behaviour.

Measurements were performed with ${ }^{88} \mathrm{Y}$ and ${ }^{22} \mathrm{Na}$ gamma sources for the three full scale values. Experimental data were then fitted with the PhResp[4] code simulations (figure 4), or by experimental data obtained with analogical acquisition (figures 5 and 6). The energy resolution is very poor for a full scale value of $1 \mathrm{~V}$. It increases when the full scale value decreases but maximum energy resolution is achieved for a full scale of $0.5 \mathrm{~V}$. The energy resolution is limited by the performance of the card (8bits). However first Compton edge of ${ }^{88} \mathrm{Y}(898 \mathrm{keV})$ is invisible for a full scale of $1 . \mathrm{V}$, appears for a full scale of $0.5 \mathrm{~V}$ and can be used as calibration point for full scale of $0.2 \mathrm{~V}$.

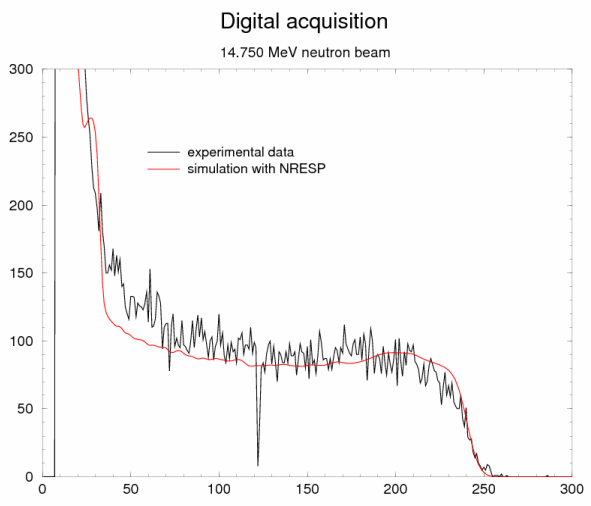

Figure 3: Recoil proton energy distribution obtained with 14.75 MeV neutron beam and compared with NRESP calculation.

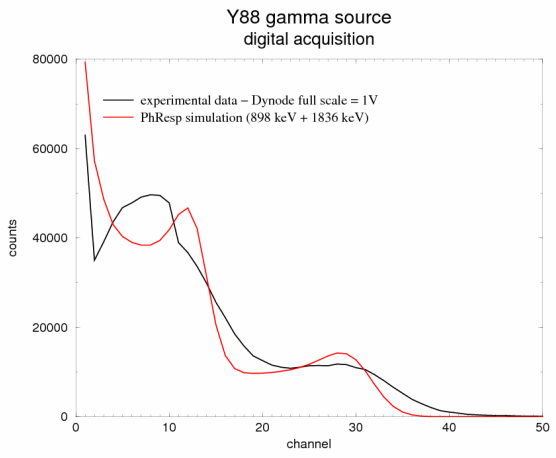

Figure 4: Compton electron energy distribution obtain with ${ }^{88} \mathrm{Y}$ gamma sources, comparison with PhResp calculation. 

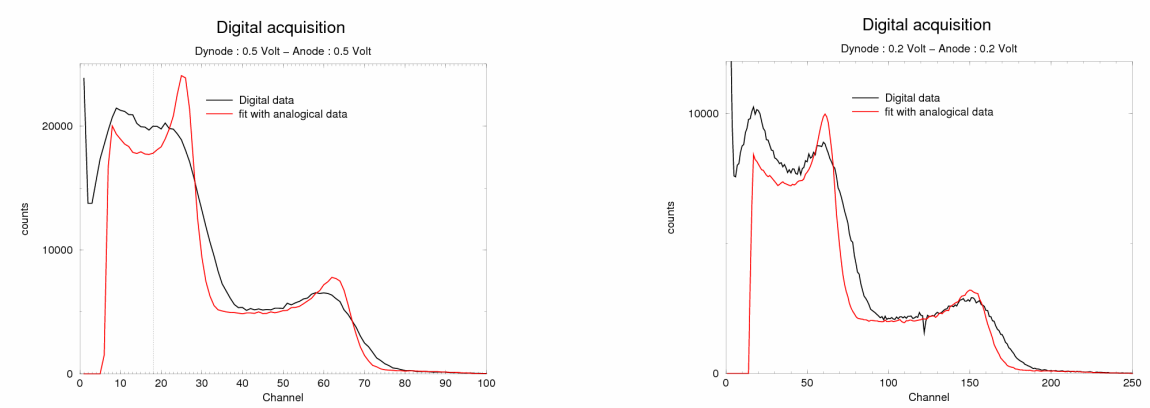

Figure 5 and 6: Compton electron energy distributions of ${ }^{88} \mathrm{Y}$ gamma source for a full scale of 0.5 Volt and 0.2 Volt, fitted by similar distribution obtained with analogical process.

\section{Conclusions and perspectives}

Prototype of digital acquisition using digital card from the market was tested in real condition in February 2006 with neutron beams produced at the AMANDE facility and with gamma sources. The discrimination process using Fourier Transform method was satisfying and discrimination threshold was comparable to the one obtained usually with analogical process.

The energy resolution for high recoil proton energies (high pulse height) was comparable to the one obtained by analogical way. But analysis of Compton electron energy distribution $\left({ }^{88} \mathrm{Y}\right)$ showed the deterioration of the energy resolution when the pulse height of dynode pulse decreases. The energy resolution obtained with analogical process was never achieved with digital acquisition system. Further studies will be done to improve this resolution. Frequency filters will be applied to reduce the noise and a theoretical function to fit the dynode pulse will be tested. Nevertheless, a new Acqiris card with 10 bits resolution is now on the market and should lead to the same resolution than that one obtained with analogical DAQ system.

The energy range, for which this acquisition system can be used, was limited by the poor choice of full scale values offered by the digital card. For neutron energy up to $20 \mathrm{MeV}$ calibration with gamma sources was not possible. As an alternative, the calibration could be performed, for neutron energy up to $15 \mathrm{MeV}$, with gamma energy higher than $1 \mathrm{MeV}$. The best neutron energy range was between $1 \mathrm{MeV}$ and $10 \mathrm{MeV}$ with this digital acquisition system. But the new 10 bits resolution card should improve the ratio pulse over noise and extend this energy range.

In conclusion tests performed with the digital acquisition prototype is quite encouraging. The use of the new 10 bits card version should improve appreciably the energy resolution and the energy detection threshold.

Next step will be devoted to study the time performances obtained with time stamping, after some software corrections. 


\section{Acknowledgments}

The authors wish to thank Kai Tittelmeier from PTB staff for explanation of high voltage feedback and valuable discussions. Acknowledgments are also addressed to the CRIIP staff for the development of the high voltage feedback unit.

\section{References}

[1] V. Gressier et al, IRSN report - SDE/2004-08, 2004

[2] D. Schlegel, "Target user's manual”, PTB report 6.41-98-1, 1998

[3] G. Dietze und H. Klein, NRESP4 and NEFF4, PTB report ISSN 0572-7170, 1982

[4] T. Novotny et al., Nuclear Instruments and Methods A, 40 (1997) 356

[5] B. Duponchel, Mémoire de projet industriel CEFIPA, 2002

[6] V. Maury. Mémoire d'ingénieur CNAM, 2004

[7] V. Gressier et al., Radiation Protection Dosimetry, 110 (1-4), p $49-52$ (2004). 